\title{
Valoración Económica del Servicio de Provisión Hídrica de la Microcuenca del río Chimborazo
}

\section{Economic valuation of the water supply service of the Chimborazo river Micro- basin}

\author{
Byron Santiago Once-Collaguazo ${ }^{l *}$, María Fernanda Rivera-Velásquez ${ }^{I}$, Carlos Wladimir \\ Izurieta-Recalde $\mathbb{B D}^{l}$ \\ ${ }^{I}$ Facultad de Ingeniería, Universidad Nacional de Chimborazo, Riobamba, Ecuador, 060150; \\ mariaf.rivera@espoch.edu.ec; cizurieta@unach.edu.ec \\ * Correspondencia: oncebyron@gmail.com
}

Recibido 28 abril 2019; Aceptado 27 mayo 2019; Publicado 06 junio 2019

Resumen:

La microcuenca del río Chimborazo, de la Parroquia San Juan, Cantón Riobamba, Provincia de Chimborazo, es la principal productora y abastecedora del recurso hídrico para las comunidades asentadas en sus alrededores, que son alrededor de 5425 hab. En la actualidad, el pago que se realiza por la distribución del servicio de agua no internaliza los aspectos ambientales para la protección de la zona de importancia hídrica y la conservación de la biodiversidad. En este sentido, en esta investigación se presentan los resultados de la valoración económica del servicio de provisión hídrica, desde una adaptación de las metodologías de Barrantes y Villavicencio, aplicada a un sistema de páramo, donde se estimó un valor real igual a $\$ 0.26 / \mathrm{m}^{3}$. El valor real fue calculado a través de la sumatoria del valor de captación $\left(\$ 0.114 / \mathrm{m}^{3}\right)$, del valor de protección $\left(\$ 1.33 \times 10^{-6} / \mathrm{m}^{3}\right)$, del valor de recuperación $\left(\$ 9.3 \times 10^{-2} / \mathrm{m}^{3}\right)$, del valor del agua como insumo a la producción $\left(\$ 1.19 \times 10^{-4}\right.$ ha $\left./ \mathrm{m}^{3}\right)$; y de los costos operativos y de administración $\left(\$ 0.054 / \mathrm{m}^{3}\right)$. La implementación de un reajuste tarifario considerando el valor real generaría mecanismos de compensación para la conservación y protección del servicio ambiental hídrico en la zona de estudio.

Palabras clave: Producción Hídrica, Reajuste Económico, Servicios Ecosistémicos, Valoración económica

Abstract: $\quad$ The Chimborazo River micro-basin in the Parroquia of San Juan of the canton of Riobamba, Chimborazo Province, is the main water resource's producer and supplier for the communities of that area, with a population of about 5425 inhabitants. At present, the payment for the distribution of the water service does not internalize environmental aspects for the protection of an area of hydric importance and for the conservation of biodiversity. In this framework, this research presents the results of the economic valuation of the water supply service, adapting the methodologies of Barrantes and Villavicencio for application to a páramo system, leading to a real estimation of $\$ 0.26 / \mathrm{m}^{3}$. This value was calculated adding the acquisition value $\left(\$ 0.114 / \mathrm{m}^{3}\right)$, the protection value $\left(\$ 1.33 \times 10^{-6} / \mathrm{m}^{3}\right)$, the recovery value $\left(\$ 9.3 \times 10^{-2} / \mathrm{m}^{3}\right)$, the water value as input to production $(\$ 1.19 \times 10$ $\left.4 \mathrm{ha} / \mathrm{m}^{3}\right)$; and the operating and administrative costs $\left(\$ 0.054 / \mathrm{m}^{3}\right)$. The implementation of a tariff readjustment considering the real value would generate compensation mechanisms for the conservation and protection of the environmental water service in the studied area.

Keywords: $\quad$ Water Production, Economic Readjustment, Ecosystem Services, Economic valuation 


\section{Introducción}

La Asamblea General de la Organización de las Naciones Unidas trabaja en la Agenda 2030 para el Desarrollo Sostenible, donde el objetivo 6 contempla acciones para garantizar la disponibilidad de agua y su gestión sostenible y el saneamiento para todos (CEPAL, 2016). Si bien, el agua es un recurso fundamental para el desarrollo de los ecosistemas terrestres, la calidad y cantidad de este recurso depende de un manejo integral de la zona de recarga.

En el quehacer mundial, se debe entender por desarrollo aquel proceso que, favorezca al progreso de un país o de una región, que contribuya a la mejora de la economía, que genere políticas de buen vivir en la población y que respete el equilibrio de los ecosistemas, contribuyendo en la medida de lo posible a mitigar los daños ya causados.

En este sentido nace la "Economía Ambiental" como una disciplina que se sustenta en valores y principios de la economía neoclásica pero que, además, trata de integrar en el modelo tradicional variables que permitan mitigar los efectos no deseados de la actividad económica denominadas externalidades. En este contexto, nace la internalización de externalidades ambientales, que tiene por objetivo internalizar, a través de los precios de mercado, aquellas externalidades ambientales negativas. (Dinar, 2003.)

Por tal razón, la valoración económica del recurso hídrico es importante para estimar los costes para la gestión integral del agua, en términos de cantidad y calidad en función del tiempo. No está por demás reflexionar que al ambiente le cuesta producir un bien y servicio, el no valorar este tipo de recursos ecosistémicos es equivalente a considéralos gratuitos y de poca importancia.

En este ámbito, se han generado varias metodologías cuyo objetivo es asignar un valor económico al agua, utilizando variables propias de la zona, asociadas al uso del recurso hídrico. (Villavicencio Aldaz, 2008), (Barrantes Moreno, 2010). Entre las estrategias para alcanzar la sustentabilidad, económica ambiental en el presente milenio, la valoración económica y la generación de mercados juega un papel preponderante que permite compensar a la naturaleza por los servicios brindados.

En Latinoamérica varias investigaciones realizadas, con especial énfasis al recurso agua, (Barrantes \& Vega, 2002), (Nuñez Parrado, 2004), (European Comision, 2008), han evidenciado la subvaloración del recurso ambiental, exponiéndoles a procesos de degradación del ambiente cuyos efectos asociados en muchos de los casos son irreversibles.

En Ecuador, las principales experiencias de valoración económica se encuentran en los Cantones de Loja, Cuenca y Bolívar (Villavicencio Aldaz, 2008; Monar Gavilánez et al., 2017) cuyos resultados han dado la pauta para la valoración de bienes y servicios ambientales que contribuyen a la generación de políticas públicas encaminadas a la conservación de las unidades hídricas.

En el proyecto Manejo de los Recursos Naturales de Chimborazo (Red consultora socio ambiental TZEDAKA Cia. Ltda, 2013) se establece la importancia de realizar acciones de salvaguardia de los recursos naturales para contribuir a la sustentabilidad ambiental. Este análisis se realiza teniendo en cuenta los pisos altitudinales, que en el recorrido del agua generan conflictos entre oferentes y demandantes, por la calidad y cantidad del recurso hídrico, siendo los más afectados las comunidades ubicadas en la parte baja donde existe mayor actividad económica. Además, se recalca la importancia de crear propuestas técnicas políticas que contribuyan de una forma significativa a la mitigación estos problemas.

Dentro de este contexto, el presente trabajo es una adaptación metodológica desde las investigaciones de Barrantes y Villavicencio (Barrantes Moreno, 2010; Villavicencio Aldaz, 2008), para la estimación de un costo real del agua, para lo cual, se consideran variables económicas ambientales provenientes de información primaria y secundaria. Los resultados evidencian que en la actualidad la base imponible es de $\$ 0.024 / \mathrm{m}^{3}$ de agua, que en relación al costo real calculado de $\$ 0.26 / \mathrm{m}^{3}$, es solamente un $10 \%$ del valor útil en términos de sustentabilidad hídrica.

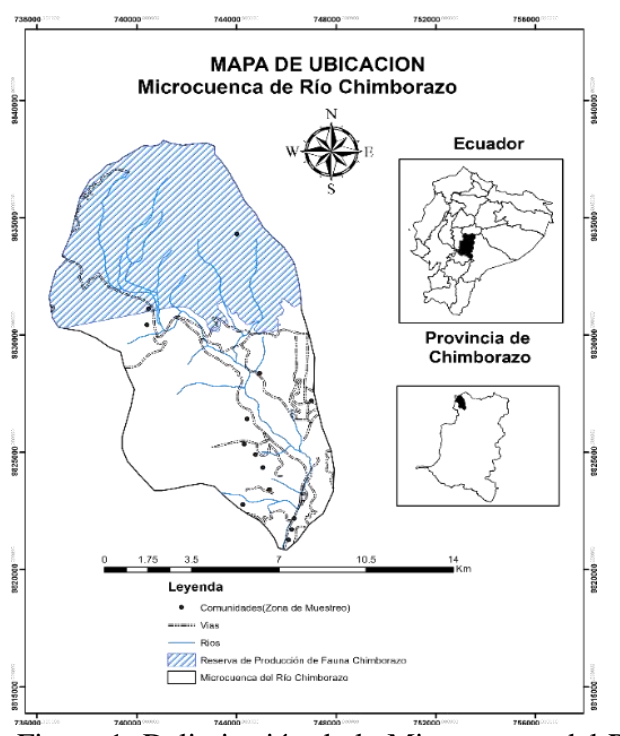

Figura 1: Delimitación de la Microcuenca del Rio Chimborazo. 


\section{Metodología}

\subsection{Descripción del lugar}

En la provincia de Chimborazo, en las parroquias de San Juan y Calpi del cantón Riobamba, y en la parroquia San Andrés del cantón Guano, se encuentra localizada la microcuenca del Rio Chimborazo con un área total 12162 ha y con una pendiente de $37 \%$. La microcuenca se encuentra divida en 3 zonas, la zona alta con 9494.50 ha y 946 habitantes; la zona media con 2563 ha y 2736 habitantes y la zona baja con 104.50 ha y 1743 habitantes. Por otro lado, posee un factor forma de $0.36 \mathrm{Ff}$, es decir, se considera alargada con una anchura promedio de $6.77 \mathrm{Km}$ y una longitud $18.56 \mathrm{Km}$.

Desde un punto de vista altitudinal se ubica a una altura de $3260 \mathrm{msnm}$ que es el lugar de descarga de las aguas al Rio Calera, y 6310 msnm, que es el punto más alto, correspondiente a la cumbre del nevado Chimborazo. El $68 \%$ de la microcuenca está ocupada por bosques y páramos, el 23\% del suelo es destinado para pastos y cultivos y solamente el $1.28 \%$ es considerado una zona de producción agrícola donde se cultiva: papa, maíz, cebada, arveja, cebolla, entre otros (Red consultora socio ambiental TZEDAKA Cia. Ltda, 2013)

El agua retenida en los páramos cubiertos por pajonal recibe 10.8 millones de metros cúbicos de agua, con una retención del 45\%; seguidos por los páramos de almohadilla que recibe 2.5 millones de metros cúbicos con una retención de humedad del $89.5 \%$ y finalmente, las plantaciones forestales reciben de 21 millones de metros cúbicos una retención del $65 \%$. De manera global el volumen total retenido, que para el caso en estudio corresponde al coste usado como valor de agua disponible $\left(V_{a}\right)$, es de $13930290.8 \mathrm{~m}^{3}$.

\subsection{Valoración económica del servicio de provisión hídrica}

La metodología utilizada tiene como base los estudios de Barrantes y Villavicencio (Barrantes Moreno, 2010; Villavicencio Aldaz, 2008), cuyos valores input para la estimación de la valoración económica del servicio de provisión hídrica, o también como valor real del agua $\left(V_{R}\right)$, mostrada en la Ec, 1, son datos propios obtenidos desde la zona de estudio. Tales técnicas tienen como objetivo servir de insumo para la generación de Planes de Compensación Ambiental, a través de políticas, programas y proyectos, que permitan modificar la idea colectiva del agua como un servicio gratuito, cuantificando un valor lo más aproximado al real. El cálculo de $\left(V_{R}\right)$, viene generado mediante la sumatoria de: el valor de captación (Ec. 2), valor de protección (Ec. 3), valor de recuperación (Ec. 4), valor de agua como insumo para la producción (Ec. 5), costos operativos y administrativos (Ec. 6). Este procedimiento permite internalizar las variables ambientales, dentro de un análisis coste-beneficio, para un posterior reajuste dentro de la planilla de pago a la población.

$$
V_{R}=V_{C}+V_{P}+V_{R E}+V_{P A}+V_{C O A}
$$

Dónde:

$V_{R}=$ valor total del servicio de producción hídrica $\left(\$ / \mathrm{m}^{3}\right)$.

$V_{C}=$ valor de captación $\left(\$ / \mathrm{m}^{3}\right)$.

$V_{P}=$ valor de protección $\left(\$ / \mathrm{m}^{3}\right)$.

$V_{R E}=$ valor de recuperación $\left(\$ / \mathrm{m}^{3}\right)$.

$V_{P A}=$ valor del agua como insumo a la producción $\left(\$ / \mathrm{m}^{3}\right)$.

$V_{C O A}=$ costos operativos y administrativos $\left(\$ / \mathrm{m}^{3}\right)$.

Las diferentes variables económicas - ambientales usadas en la Ec. 1 se detallan a continuación:

Valor de Captación y/o Productividad Hídrica $\left(V_{C}\right)$.

La Ec. 2 determina el valor de captación o productividad hídrica $\left(V_{C}\right)$, estimado a través del: 1) el costo de oportunidad $\left(C_{o}\right)$ y 2$)$ la importancia de la cubierta vegetal protectora (\&). En el primer caso, corresponde a un valor monetario asociado con la actividad económica que compite con el ecosistema páramo, y es generado mediante el levantamiento de información primara, es decir, en el caso de estudio se realizó mediante una encuesta, como se describe en la sección 2.3. Esta fase permite identificar la actividad económica más representativa, de esta manera se obtienen los costos totales de ingresos y egresos al año. La información levantada reflejará a ciencia cierta el costo de oportunidad, componente necesario para encontrar la variable $\left(V_{C}\right)$. Para el segundo caso, se estima $\&$, que varía entre 0 y 1 , valor generado a través de la percepción social, que la clasifica en niveles jerárquicos, donde se ve reflejada la importancia de la cubierta para la protección del recurso hídrico.

$$
V_{C}=\frac{\& \cdot C_{O} \cdot A b i}{V_{a}}
$$

Dónde:

$V_{C}=$ valor de captación o productividad hídrica por la cubierta vegetal $\left(\$ / \mathrm{m}^{3}\right)$. 
$\&=$ importancia de la cubierta vegetal protectora (adimensional).

$C_{O}=$ costo de oportunidad ( $\$ /$ ha/año).

$A b i=$ área de la zona de importancia hídrica (ha). $V_{a}=$ volumen del agua disponible ( $\left.\mathrm{m}^{3} / \mathrm{año}\right)$.

Valor de Protección y el Valor de recuperación $\left(V_{P}, V_{R E}\right)$.

El valor de protección $V_{P}$ (Ec. 3) y el valor de recuperación $V_{R E}$ (Ec. 4), promueven acciones de conservación, protección y recuperación, a ser implementadas dentro de los ecosistemas de páramos, como un mecanismo de mitigación a los efectos negativos (Ramakrishna, 1997). Tales acciones generan un costo $C$ que deberá ser considerado dentro de una estructura de valoración económico-ecológica para el uso del agua.

$$
\begin{gathered}
V_{P}=\frac{\& \cdot C}{V_{a}} \\
V_{R E}=\frac{\& \cdot C_{R} \cdot \text { Arih }}{V_{a}}
\end{gathered}
$$

Dónde:

$V_{P}=$ costo de protección de la zona de importancia hídrica $\left(\$ / \mathrm{m}^{3}\right)$.

$V_{R E}=$ valor de recuperación hídrica de cuenca hidrográfica $\left(\$ / \mathrm{m}^{3}\right)$.

$C=$ costo para las actividades de protección de la cuenca (\$/ha/año).

$C_{R}=$ costo para las actividades de protección de la cuenca (\$/ha/año).

Arih =área a recuperar en la zona de importancia hídrica (ha).

Valor del agua según su uso $\left(V_{P A}\right)$

En la gran mayoría de actividades económicas, el recurso hídrico es un insumo significativo en los procesos de producción, por tal razón, es importante asignarle un precio que responda al valor de escasez de este servicio ambiental. La asignación de un costo de producción implica la utilización de diferentes técnicas, debido a la variada utilización que se hace de este recurso. El enfoque usado dentro de este estudio considera al agua como insumo de la producción (Ec. 5), criterio basado en el hecho que la zona presenta principalmente características agrícolas ganaderas.

En dependencia de las condiciones climáticas del sector, los cultivos pueden necesitar mayor o menor regadío, incrementando o disminuyendo su productividad agrícola, y esta variación identificada in situ en la producción es utilizada para calcular el valor total de agua.

$$
V_{P A}=\frac{V_{P C R}-V_{P S R}}{V}
$$

Dónde:

$V_{P A}=$ valor total del agua en la producción agrícola (\$/ha).

$V_{P C R}=$ valor de la producción agrícola con riego (\$/ha).

$V_{P S R}=$ valor de la producción agrícola sin riego (\$/ha)

$V=$ volumen de agua utilizado para la producción $\left(\mathrm{m}^{3}\right)$

Valor de los costos administrativos y de operación $\left(V_{C O A}\right)$.

El valor de los costos administrativos y de operación, calculados en la Ec. 6 toma en consideración para su cálculo los gastos de mantenimiento de la infraestructura y comprenden tanto los costos de tratamiento pre-servicio como los costos de post-servicio.

$$
V_{C O A}=T_{R}+P_{T R}
$$

Dónde:

$V_{C O A}=$ costos operativos y administrativos $\left(\$ / \mathrm{m}^{3}\right)$. $T_{R}=$ costos de tratamiento pre-servicio $(\$)$.

$P_{T R}=$ tratamiento de post-servicio (\$).

La Ec. 7 estima los costos de pre-servicio que agrupan los costos de infraestructura, insumos y mano de obra.

$$
T_{R}=\frac{C_{F}+C_{I}+M_{O}}{V_{a}}
$$

Dónde:

$T_{R}=$ costos de tratamiento pre-servicio (\$).

$C_{F}=$ costos de infraestructura (\$).

$C_{I}=$ costos en insumos $(\$)$.

$M_{O}=$ costos en mano de obra (\$)

El $P_{T R}$ es el rubro de tratamiento post-servicio o costos de depreciación del agua que comprende el costo por traslado de aguas residuales y su posterior tratamiento en metros cúbicos.

\subsection{Levantamiento de información base}

El método de levantamiento de información primaria se realizó mediante el desarrollo de un taller participativo, a las comunidades asentadas en el área de estudio, a una población de $\mathrm{N}=5425$ habitantes mediante la aplicación de una encuesta. 
El tamaño de la muestra se calculó en función de una población conocida (Ec. 8), teniendo como valor representativo 71 unidades (Morillas, 2010):

$$
n=\frac{N \cdot Z_{\alpha}^{2} \cdot p \cdot q}{d^{2}(N-1)+Z_{\alpha}^{2} \cdot p \cdot q}
$$

Donde:

$Z=$ Nivel de confianza (1.94)

$\mathrm{P}=$ Variable de investigación que participa directamente (0.05)

$\mathrm{q}=$ Variable de investigación que participa incipientemente (1-p, 0.90).

$\mathrm{d}=$ Nivel de precisión $(10 \%)$.

La encuesta tuvo por objetivo determinar $C_{O} \mathrm{y} \&$, estas variables fueron estimadas mediante preguntas semiestructuradas con la finalidad de generar valores monetarios en dólares de:

- $\quad$ Arriendo de hectáreas de potrero disponible.

- Comercialización de los productos agropecuarios.

- $\quad$ Pago por mano de obra a jornaleros.

- Insumos para la producción agropecuaria.

- Importancia de la cobertura vegetal.

- Actividades productivas, entre otros.

Adicionalmente, a través de la encuesta se obtuvieron datos de las sentencias de caudal en $\mathrm{m}^{3}$ /año asignados a cada una de las comunidades como se muestra en la tabla 1 .

Tabla 1: Caudales asignados a las comunidades asentadas en la microcuenca del Rio Chimborazo.

\begin{tabular}{|c|c|c|c|}
\hline Nro & Sistema & $\begin{array}{c}\text { Familia } \\
\mathrm{s}\end{array}$ & $\mathbf{m}^{3 /} \mathbf{a} \tilde{\mathbf{n o}}$ \\
\hline 1 & Comunidad Chimborazo & 120 & 31536.00 \\
\hline 2 & Asociación Pasguazo & 20 & 63072.00 \\
\hline 3 & Comunidad La Delicia & 67 & 189216.00 \\
\hline 4 & Comunidad Ganquiz & 30 & 37843.20 \\
\hline 5 & Comunidad Santa Isabel & 60 & 15768.00 \\
\hline 6 & $\begin{array}{l}\text { Asociación Cachipamba } \\
\text { Comunidad Santa Teresita }\end{array}$ & 33 & 31536.00 \\
\hline 7 & de Guabug & 166 & 15768.00 \\
\hline 8 & Comunidad Shobol Pamba & 77 & 31536.00 \\
\hline 9 & $\begin{array}{l}\text { Comunidad Guadalupe } \\
\text { Cooperativa Santa Teresita }\end{array}$ & 37 & 6307.20 \\
\hline 10 & de Guabug & 45 & 63072.00 \\
\hline 11 & $\begin{array}{l}\text { Comunidad Calera Grande } \\
\text { Comunidad Calerita Santa }\end{array}$ & 238 & 26805.60 \\
\hline 12 & Rosa & 55 & 19236.96 \\
\hline 13 & $\begin{array}{l}\text { Comunidad Pisicaz } \\
\text { Comunidad Tambo }\end{array}$ & 78 & 2838.24 \\
\hline 14 & $\begin{array}{l}\text { Huasha } \\
\text { Asociación Chorrera }\end{array}$ & 48 & 110376.00 \\
\hline 15 & $\begin{array}{l}\text { Mirador } \\
\text { Comunidad Shobol }\end{array}$ & 23 & 47304.00 \\
\hline 16 & $\begin{array}{l}\text { Llinllín } \\
\text { Barrio San Antonio de }\end{array}$ & 156 & 126144.00 \\
\hline \multirow[t]{2}{*}{17} & Rumipamba & 66 & 50457.60 \\
\hline & TOTAL & 1319 & 868816.80 \\
\hline
\end{tabular}

\section{Resultados y Discusión}

La zona de estudio presenta características especiales como son: ecológicas, edafológicas, geomorfológicas y climáticas, que la convierten en un ecosistema fundamental para la regulación hídrica. Considerando la zona de páramo aquella productora de agua, o zona de interés hídrico que tiene una área total de $69.1 \%$, se estimó un costo real, es decir un valor económico y ecológico, del servicio de agua.

La presente investigación se implementó en dos fases. Para el desarrollo de la primera fase de levantamiento de información in situ se consideraron 17 comunidades, donde se encuentran asentadas 1.319 familias, con una población aproximada de 5.425 personas, como se muestra en la tabla 1.

El levantamiento de la información primaria tuvo como finalidad dar valores numéricos a las variables económicas para el cálculo del $V_{C}, V_{P}$, $V_{R} y V_{C O A}$, mostradas en la Ec, 2. Ec. 3, Ec. 4 y Ec. 6 respectivamente. $\mathrm{La}$ variable \& que representa la importancia de la cobertura vegetal protectora en el control de volúmenes de escorrentía, retención y generación de agua en términos de calidad y cantidad para los usuarios del servicio. El valor obtenido de \& es de 0.83 que cualitativamente se podría interpretar como muy importante. La ponderación \& se obtuvo mediante entrevistas semiestructuradas en relación al servicio jerarquizándolo en los siguientes niveles: poca importancia, mediana importancia, y mucha importancia. (Villavicencio Aldaz, 2008). Adicionalmente, a través del levantamiento de información in situ fue determinado el costo de oportunidad $C_{o}$ con un valor de $\$ 228$ ha/año.

El Abi es el área de importancia hídrica que tiene un total de 8.327 ha., cuyo valor fue calculado sumando las áreas de páramos de almohadilla y paramos de pajonal, consideradas las zonas de interés hídrico. El valor de $V_{a}$ disponible es igual a 13.930.290,80 $\quad \mathrm{m}^{3} / \mathrm{año.} \mathrm{El} \mathrm{Arih} \mathrm{es} \mathrm{el} \mathrm{área} \mathrm{a}$ recuperar en la zona de importancia hídrica, cuyo valor es igual a $2.897 \mathrm{Ha}$. Los datos de Abi, Arih y $V_{a}$ fueron tomados del Plan de Manejo y Cogestión de la Microcuenca del río Chimborazo.

La variable $V_{P}$, con un valor de $\$ 1,33 \times 10^{-6} / \mathrm{m}^{3}$, corresponde a la inversión en términos monetarios para las actividades de protección de la microcuenca y comprende: el mantenimiento y la protección del área de interés hídrico a través de programas de vigilancia comunitaria y educación 


\section{Unach}

ambiental. La variable $V_{P R}$ igual a $\$ 0.093 / \mathrm{m}^{3}$, se estimó a través de los costos de recuperación como son: la creación de un vivero comunal y la reforestación con plantas nativas en la zona.

Para el cálculo de $V_{P A}$ mostrado en Ec. 5 se calculó $V_{P C R}$ y $V_{P S R}$, que tiene valores de $\$ 3.454 .38$ /ha para la producción con riego y $\$ 2.768,32$ /ha para la producción sin riego, respectivamente. En esta estimación se consideró, los costos de producción, rendimiento y precio del cultivo de Solanum tuberosum, conocida comúnmente como papa en la zona de Ecuador, por ser el cultivo de mayor producción agrícola en el sitio de estudio. El parámetro $V$ es considerado el volumen de agua utilizado en el riego que tiene un valor igual a $4.365 .000 \mathrm{~m}^{3}$.

Para el cálculo de $T_{R}$, mostrada en Ec. 7, que representa los costos de tratamiento en el preservicio se toma en consideración las variables $C_{F}=\$ 395.700, C_{I}=\$ 15.500$ y $M_{O}=\$ 12.400$ como costos de infraestructura, costos de insumos y mano de obra, respectivamente.

Adicionalmente, al costo $T_{R}$ se le suma el costo de post-servicio que corresponde a la tarifa de consumo impuesta por la empresa municipal prestadora del servicio que es de $\$ 0,024 / \mathrm{m}^{3}$ consumo de agua potable de la empresa que presta el servicio

La tabla 2 evidencia los costos de internalización del del agua, es decir, que lo que en un principio era considerado un coste externo para la producción se transforma en un coste interno a ser asumido por los usuarios que se benefician del recurso.

Tabla 2: Costo real del agua a partir de la suma de las variables económica utilizadas.

\begin{tabular}{cc}
$\begin{array}{c}\text { Variables } \\
\text { económicas }\end{array}$ & Valor $\left(\$ / \mathrm{m}^{3}\right)$ \\
\hline$V_{C}$ & $1.1 \times 10^{-1}$ \\
$V_{P}$ & $1.33 \times 10^{-6}$ \\
$V_{R E}$ & $9.31 \times 10^{-2}$ \\
$V_{P A}$ & $1.57 \times 10^{-4}$ \\
$V_{C O A}$ & $5.4 \times 10^{-2}$ \\
$\boldsymbol{V}_{\boldsymbol{R}}$ & $\mathbf{2 . 6 \times 1 0 ^ { - 1 }}$ \\
\hline
\end{tabular}

La tabla 2 muestra el $V_{R}=\$ 2.6 \times 10^{-1} / \mathrm{m}^{3}$ considerado el costo real del agua a partir de la suma de las variables económica utilizadas. Este valor, comparado con otros estudios en diferentes zonas de Ecuador, no difieren significativamente por ejemplo: en la microcuenca Curitroje calcula un $V_{R}=\$ 2,9 \times 10^{-2} / \mathrm{m}^{3}$ (Maza, 2002); en la microcuenca El Salado se obtiene un $V_{R}=\$ 2,7 \mathrm{x}$ $10^{-1} / \mathrm{m}^{3}$ (Hernández Ocampo \& Reyes Sánchez, 2007); en la microcuenca Chorera -Tinajones estima un $V_{R}=$ de $\$ 2,2 \times 10^{-1} / \mathrm{m}^{3}$ y en la microcuenca El Limón obtuvo un $V_{R}=\$ 1,6 \times 10$ ${ }^{-1} / \mathrm{m}^{3}$ (Coronel, 2005).

Si se realizará un ajuste tarifario a los usuarios del servicio en el consumo de agua usando un $V_{R}=$ $\$ 2,6 \times 10^{-1} / \mathrm{m}^{3}$, se generaría cerca de $\$ 225.892,37$ /año, que correspondería aproximadamente a un pago mensual por familia de $\$ 14,3$, es decir este valor serviría para la conservación de la microcuenca del río Chimborazo.

Si bien es cierto, el agua es importante para la realización de actividades cotidianas, en el pensamiento colectivo de las comunidades, es concebida como un recurso gratuito de poco, o ningún valor monetario, es decir, desde un punto de vista económico, no existe un mercado para la prestación del servicio. Esto provoca la degradación del ecosistema presente en la microcuenca, alejándose de los objetivos de desarrollo sustentable que considera una distribución equitativa y socialmente justa de este recurso. La conservación de los páramos debe ser realizada en su estado natural, es decir, el cuidado de sus bienes y servicios ecosistémicos (Díaz, 2005).

En este sentido, la estimación del $V_{R}$ se puede considerar un criterio económico para un reajuste de pago por servicios ambientales, tal valor puede convertirse en un instrumento valioso para la toma de decisiones en favor del desarrollo de la microcuenca, y a la vez garantizar un flujo sostenible del servicio ambiental hídrico.

\section{Conclusiones}

La comunidades asentadas, en un área total de 12162 ha, dentro de la microcuenca del Rio Chimborazo se desarrollan en un ecosistema que presenta las siguientes características: $68 \%$ páramo, $23 \%$ suelos destinados a pastos y cultivos $\mathrm{y}$, el $1.28 \%$ es una zona destinada para la producción agrícola, estas particularidades hacen que este ecosistema cumpla un rol de regulador social y económico para sus habitantes. Por tal razón, es importante que cada uno de ellos identifique los bienes y servicios ecosistémicos presentes en la zona, y que sea consciente de las actividades que puede, o no, ejecutar sobre él y como estas pueden contribuir a su cuidado o degradación. 


\section{Unach}

La zona de importancia hídrica que corresponde al $68 \%$ del total de la microcuenca retiene un volumen total de $13.930 .290,80 \mathrm{~m}^{3}$. Por razón, la compensación económica al ecosistema de páramo, por parte de los usuarios que se benefician del recurso hídrico, es fundamental para la creación de planes y programas que garanticen las sostenibilidad del ecosistema para las futuras generaciones. En este sentido, y bajo la premisa que en la actualidad las comunidades solo costean el pago de pre -servicio, sin internalizar sus externalidades (variables económicasambientales), se valoró el servicio ambiental hídrico a través de la estimación de un valor real del agua $V_{R}$ igual a $\$ 0,26 / \mathrm{m}^{3}$. La implementación de este valor económico, a la tarifa mensual, contribuiría a proteger, conservar y recuperar la microcuenca.

\section{Conflicto de Intereses}

Los autores declaramos que no existe conflicto de intereses dentro de la investigación o con personas o instituciones públicas y privadas.

\section{Referencias}

Barrantes Moreno, G. (2010). Evaluación del servicio ambiental hídrico en la cuenca del río Tempisque y su aplicación al ajuste de tarifas. Bucaramanga: LEBRET. Recuperado de https://dialnet.unirioja.es/descarga/articu lo/5983176.pdf

Barrantes, G. \& Vega, M. (2002). Evaluación del servicio ambiental hídrico en la cuenca del río Tempisque y su aplicación al ajuste de tarifas. ASOTEMPISQUE.

CEPAL. (2016). Agenda 2030 y los Objetivos de Desarrollo Sostenible. Una oportunidad para América Latina y el Caribe. Santiago: Naciones Unidas. Recuperado de

http://www.sela.org/media/2262361/age nda-2030-y-los-objetivos-de-desarrollosostenible.pdf

Coronel, R. J. (2005). Valoracion Economica del servicio ambiental hídrico de la microcuenca hidrológica el Limón. 225.

Díaz, M. (2005). Páramos Hidrositemas Sensibles. Revista de Ingenieria - Universidad de los Andes, 12

Dinar , A. (2003.). The political economy of water pricing reforms (Chinese). Washington, DC: World Bank. Recuperado de http://documents.worldbank.org/curated/ en/493881468769727386/The-politicaleconomy-of-water-pricing-reforms

European Comision. (2008). The Economist of ecosystems and byodiversity. Germany: Welzel+Hardt, Wesseling. Recuperado de

http://ec.europa.eu/environment/nature/b iodiversity/economics/pdf/teeb_report.pd $\mathrm{f}$

Hernández Ocampo , F., \& Reyes Sánchez, C. (2007). Valoración económica y ecológica del recurso hídrico para establecer un pago por servicio ambiental en la microcuenca "El Salado”. Universidad Nacional de Loja, Loja. Recuperado de http://dspace.unl.edu.ec/jspui/bitstream/1 23456789/5878/1/VALORACI\%C3\%93 N\%20ECON\%C3\%93MICA\%20Y\%20 ECOL\%C3\%93GICA\%20DEL\%20REC URSO $\% 20 \mathrm{H} \% \mathrm{C} 3 \% 8 \mathrm{DDRICO} . \mathrm{pdf}$

Maza, B. (2002). Valoración económica ecológica del agua de la microcuenca hidrográfica Curitroje. 144

Monar Gavilánez, N., González Rivera, M., González Rivera, V., Saltos Espín, R., Chávez Coloma, L. \& Fierro Borja, S. (2017). Valoración económica de los bienes y servicios ambientales en la microcuenca del río Illangama. Revista Ecuatoriana de Investigaciones Agropecuarias, 1-7.

Morillas, A. (2010). Muestreo en poblaciones finitas. Recuperado de https://www.ucursos.cl/ingenieria/2010/1/IN3401/1/ma terial_docente/bajar?id_material=280296

Nuñez Parrado, D. V. (2004). Valoración económica del serivicio ecosistémico de producción del agua, del bosque de la cuenca Llancahue, Décima región. Valdivia-Chile: Universidad Austral de Chile. Universidad Austral de Chile, Valdivia - Chile. Recuperado de http://cybertesis.uach.cl/tesis/uach/2004/ egn973v/pdf/egn973v.pdf

Ramakrishna, B. (1997). Estrategia de Extensión para el Manejo Integrado de Cuencas Hidrográficas: $\quad$ Conceptos $y$ Experiencias. San José: GTZ. Recuperado de http://www.infocuencas.com/img/kcfind er/files/estrategias $\% 20 \mathrm{de} \% 20$ extension $\% 20$ para\%20el\%20manejo\%20integrado $\% 20$ de\%20cuencas.pdf 


\section{Unach}

Red consultora socio ambiental TZEDAKA Cia.

Ltda. (2013). Plan de manejo y

Coogesatión de la microcuenca del Río

Chimborazo. Gobierno Autónomo

Descentralizado de la Provincia de

Chimborazo, Riobamba. Recuperado de http://www.fao.org/forestry/45914-

060f46ef0d5931a02bf22fd97cd987a31.p df

Villavicencio Aldaz, C. A. (2008). Valoracion Socioeconomica y Ambiental del recurso hidrico de la microcuenca de Atacuí, Parroquia Santiago, Canton Loja. Universidad Nacional de Loja, Loja. Recuperado de http://dspace.unl.edu.ec/jspui/handle/123 $456789 / 5708$ 